\title{
In overweight/obese but not in normal-weight women, polycystic ovary syndrome is associated with elevated liver enzymes compared to controls
}

\author{
Frangiskos Economou ${ }^{1,2}$, Xenofon Xyrafis ${ }^{1,2}$, Sarantis Livadas ${ }^{1,2}$, Ioannis I. Androulakis ${ }^{3}$, \\ Georgia Argyrakopoulou ${ }^{2}$, Charikleia D.Christakou ${ }^{1,2}$, Eleni Kandaraki ${ }^{4}$, Eleni Palioura ${ }^{1}$, \\ Evanthia Diamanti-Kandarakis ${ }^{1,2}$
}

${ }^{1}$ Endocrine Section, First Department of Internal Medicine, Laiko General Hospital, Medical School, University of Athens, ${ }^{2}$ PCOS Clinic, Eugenideio Hospital, Medical School, University of Athens, ${ }^{3}$ Department of Endocrinology and Diabetes Center, General Hospital of Athens “G. Genimatas", Athens, Greece, ${ }^{4}$ Hull Royal Infirmary Hospitals, UK

\begin{abstract}
OBJECTIVE: To investigate liver enzymes in a cohort of women with Polycystic Ovary Syndrome (PCOS) and controls divided according to body mass index (BMI) and their association with features of the syndrome. DESIGN: Eighty-three PCOS women and 64 healthy women were studied. Patients and controls were subdivided into two groups, a lean subgroup (BMI $<25 \mathrm{~kg} /$ $\mathrm{m}^{2}$ ) and an overweight/obese subgroup $\left(\mathrm{BMI}>25 \mathrm{~kg} / \mathrm{m}^{2}\right)$. Clinical history, height and weight were obtained and metabolic and hormonal parameters were determined. RESULTS: Serum fasting insulin, fasting glucose, HOMA-IR, triglycerides and total cholesterol were significantly higher $(\mathbf{p}<0.05)$ in women with PCOS compared to controls. No significant difference in serum liver enzymes levels between PCOS women and controls was detected. However, serum levels of alanine aminotransferase (ALT) $(17.7 \mathrm{vs.} 14.1 \mathrm{U} / \mathrm{L}, \mathrm{p}<0.05)$ and $\gamma$-glutamyl transpeptidase $(\gamma$ GT) $(17.9$ vs. $13.4 \mathrm{U} / \mathrm{L}, \mathbf{p}<0.05)$ were significantly higher in overweight/obese PCOS women compared with overweight/obese controls. In overweight/obese PCOS patients and controls, ALT levels were positively correlated with free androgen index (FAI) $(r=0.25 p<0.05)$ and total testosterone levels $(r=0.33 p<0.01)$. CONCLUSIONS: The finding of elevated liver enzymes in overweight/obese PCOS women raises the question of screening for non-alcoholic fatty liver disease in this group.
\end{abstract}

Key words: Body Mass Index, Insulin resistance, Liver enzymes, Non-alcoholic fatty liver disease, Polycystic Ovary Syndrome 


\section{INTRODUCTION}

Polycystic ovary syndrome (PCOS) is the commonest endocrinopathy of premenopausal women ${ }^{1}$ characterized by both reproductive and metabolic abnormalities. ${ }^{2}$ Women with PCOS commonly display a clustering of metabolic abnormalities, including impaired glucose tolerance, ${ }^{3}$ insulin resistance, ${ }^{2}$ dyslipidemia, ${ }^{4}$ increased prevalence of obesity, ${ }^{5,6}$ lowgrade chronic inflammation ${ }^{7}$ and increased oxidative stress. ${ }^{8,9}$ Insulin resistance has been demonstrated in both obese and non-obese women with PCOS. ${ }^{2}$ Non-alcoholic fatty liver disease (NAFLD) has been associated with insulin resistance ${ }^{10,11}$ and obesity. ${ }^{12}$

NAFLD is characterized by the accumulation of fat in the liver in the absence of excessive alcohol consumption. NAFLD encompasses a spectrum of histological changes extending from benign simple steatosis (non-alcoholic fatty liver - NAFL) to steatohepatitis (NASH), progressive fibrosis and, ultimately, cirrhosis. ${ }^{13}$ Although NAFLD is primarily a histological diagnosis, elevated liver enzymes have been used as a noninvasive surrogate marker of NAFLD, provided that other potential causes of liver disease (chronic viral hepatitis, alcohol-induced liver disease, etc.) have been excluded. The typical pattern of abnormal liver biochemical profile includes increased serum aminotrasferases, with a predominant increase of alanine aminotransferase (ALT), relative to aspartate aminotransferase (AST), accompanied by elevated $\gamma$-glutamyl transpeptidase $(\gamma \mathrm{GT})$ levels. ${ }^{14,15}$ Although most patients with NASH will have elevated serum levels of transaminases, liver function tests (LFTs) may occasionally be normal.

Most intriguingly, recent research has drawn attention to the "dose-dependent", clinical significance of increasing ALT levels within the normal range. An increased ALT without overt hypertransaminasemia is a proposed independent predictor of imminent NAFLD. ${ }^{16}$ Additionally, several prospective studies have shown that increased ALT and $\gamma$-GT concentrations, still within the normal limits, predict the future development of type 2 diabetes mellitus and Cardiovascular (CV) events, independently of other known risk factors. ${ }^{17-20}$

Elevated ALT, above the level of $35 \mathrm{U} / \mathrm{lt}$, has been detected in approximately $30 \%$ of overweight/ obese women with PCOS. ${ }^{21,22}$ This prevalence rate significantly exceeds the population-based estimate for elevated aminotransferase levels ${ }^{14}$ and is further increased if stricter cutoffs are applied. ${ }^{22,24} \mathrm{~A}$ significant elevation of aminotransferase levels (ALT and/or AST above $60 \mathrm{U} / \mathrm{L}$ ) is highly likely to concur with the imaging finding of fatty infiltration in obese women with PCOS. ${ }^{22}$ Ultrasonographic evidence is more prevalent than the biochemical evidence of NAFLD in lean, overweight and obese PCOS women, ${ }^{25}$ but the use of a lower cutoff for ALT may significantly increase the sensitivity of this marker in identifying mild NAFLD. ${ }^{26}$

Overall, existing evidence of NAFLD in PCOS women is still scarce and presents certain limitations. Of the available studies, only one included a control group. ${ }^{24}$ Moreover, only one study included both lean and obese patients ${ }^{25}$ while the others either included only overweight/obese patients or did not subdivide patients according to BMI. ${ }^{21,22}$ Additionally, serum levels of $\gamma \mathrm{GT}$ were determined only in one of the available four studies. ${ }^{25}$

The aim of the present study was to investigate liver enzymes in a cohort of women with PCOS and controls divided according to $\mathrm{BMI}$ in order to explore a possible independent effect of PCOS and obesity on liver function and to uncover potential associations of serum liver enzymes with hormonal parameters and indices of insulin resistance.

\section{PATIENTS AND METHODS}

One hundred and forty-seven women of whom 83 were consecutive patients with PCOS attending the PCOS-Endocrine Unit in Eugenideion University Hospital, were studied. PCOS was defined according to the 1990 National Institutes of Health consensus criteria. ${ }^{27}$ Participants were diagnosed with PCOS if they had oligo/amenorrhea (menstrual intervals $>35$ days) combined with clinical signs of androgen excess (hirsutism, Ferriman-Gallwey score $>8$ ) and/ or elevated serum levels of total testosterone, after the exclusion of other causes of irregular menstrual cycles or androgen excess (hyperprolactinemia, uncontrolled thyroid disease, congenital adrenal hyperplasia, 
Cushing's syndrome, androgen-secreting tumor or pregnancy). Patients were subdivided into two groups according to their BMI value: the lean (BMI $<25 \mathrm{~kg}$ / $\mathrm{m}^{2}$ ) subgroup (44 patients) and the overweight/obese (BMI $\geq 25 \mathrm{~kg} / \mathrm{m}^{2}$ ) subgroup (39 patients).

A group of 64 normo-androgenemic and regularly ovulating women, confirmed by progesterone levels on three consecutive cycles, served as the control group. Controls were also subdivided into two groups according to their BMI values. The lean subgroup included 34 subjects and the overweight/obese subgroup included 30 .

Lean and overweight/obese controls did not differ in age and BMI from the corresponding lean and overweight/obese patients. The exclusion criteria were the following: 1) a history of alcohol intake higher than 20 gr per day, 2) the use of oral contraceptives, insulin sensitizers or any other known medication which might interfere with metabolic or hormonal measurements during the three months preceding the entry to the study, 3) a history of chronic viral hepatitis, hemochromatosis, autoimmune liver disease, other chronic liver disease, 4) a history of medication use causing hepatotoxicity or elevation of liver enzymes, 5) a history of type 2 diabetes mellitus or fasting glucose $\geq 126 \mathrm{mg} / \mathrm{dL}$.

Following a complete history, all women underwent clinical assessment and had anthropometric measurements (weight, height). In each subject participating in the study the following parameters were determined after an overnight fast: glucose, lipids, AST, ALT, $\gamma \mathrm{GT}$, total and free Testosterone, sex hormone binding globulin (SHBG) and free androgen index (calculated as the ratio of total testosterone levels in $\mathrm{nmol} / \mathrm{l}$ to SHBG levels in $\mathrm{nmol} / \mathrm{lt}$ ). The assays employed for endocrine and biochemical measurements have previously been reported..$^{28}$ Liver enzymes (ALT, AST) were measured by the IFCC method, NADH without P-5'-P, and $\gamma$ GT with L-Gamma-Glutamyl-3-Carboxyl-4-nitroanilide substrate by Abbott Chemistries. Insulin resistance was assessed by the HOMA-IR index as: fasting serum insulin $(\mu \mathrm{U} / \mathrm{ml}) \mathrm{x}$ fasting plasma glucose (mmol/l)/22.5. ${ }^{29}$ Informed consent was obtained from every participant in the study and the study was approved by the Ethics Committee of the Laiko general hospital.

\section{Statistical analysis}

Results are presented as mean (SD). Normality of the data was tested according to the KolmogorovSmirnov test. Differences between the PCOS and control groups were detected using the unpaired Student's t test and Mann-Whitney test as appropriate. Correlations between variables were evaluated by Pearson's and Spearman's correlation coefficients. The chi-square test was used to compare prevalence rates of different parameters among groups. Stepwise multiple regression analysis was conducted to investigate the impact of PCOS status and the parameters measured on the liver enzymes. A p value $<0.05$ was considered significant. All statistical analyses were performed using SPSS 13.0 for Windows (SPSS, Inc., Chicago, IL).

\section{RESULTS}

Women with PCOS and controls did not differ in age and BMI. The values of liver enzymes did not differ between the total group of PCOS women and controls (Table 1). The percentage of women in the entire PCOS group having elevated liver enzymes (>25 U/L) was $12 \%(10 / 83)$ for ALT and 7.2\% (6/83) for $\gamma \mathrm{GT}$, while the corresponding percentages in the control group were $4.6 \%(3 / 64)$ and $1.5 \%(1 / 64)$ $(\mathrm{p}=0.12$ and $\mathrm{p}=0.11$, respectively). Women with PCOS had higher serum levels of Testosterone (T), fTesto and Free Androgen Index (FAI), and lower levels of SHBG $(p<0.05)$. Serum fasting insulin, fasting glucose, HOMA-IR, Triglycerides (Trg) and Total Cholesterol were significantly higher $(\mathrm{p}<0.05)$ in patients than in controls (Table 1).

Lean and overweight/obese controls did not differ in age and BMI from the respective subgroup of PCOS patients. Both lean and overweight/obese women with PCOS had significantly higher total testosterone levels compared to the levels in the respective subgroups of controls ( $\mathrm{p}<0.001)$. Lean women with PCOS as compared to lean controls had significantly higher fasting glucose levels ( $\mathrm{p}<0.01)$, higher fasting insulin levels $(p<0.05)$, higher HOMA-IR $(p<0.01)$ and higher triglyceride levels $(p<0.05)$. Total cholesterol levels were not significantly different though marginally higher in lean PCOS women than in lean controls. Serum levels of liver enzymes, namely AST, ALT 
Table 1. Anthropometric, hormonal and biochemical characteristics of patients with polycystic ovary syndrome (PCOS) and controls (mean SD).

\begin{tabular}{lccc}
\hline Variable & PCOS $(\mathbf{N = 8 3})$ & $\begin{array}{c}\text { Controls } \\
(\mathbf{N}=64)\end{array}$ & $\boldsymbol{P}$ \\
\hline Age $($ years $)$ & $25.0 \pm 4.9$ & $26.6 \pm 4.0$ & $0.06^{*}$ \\
BMI $\left(\mathrm{kg} / \mathrm{m}^{2}\right)$ & $25.8 \pm 4.6$ & $24.7 \pm 4.6$ & $0.17^{* *}$ \\
Glucose $(\mathrm{mg} / \mathrm{dl})$ & $94.2 \pm 13.7$ & $82.2 \pm 12.5$ & $<0.001^{*}$ \\
Insulin $(\mu \mathrm{U} / \mathrm{ml})$ & $10.0 \pm 6.7$ & $7.5 \pm 4.3$ & $<0.05^{* *}$ \\
HOMA-IR & $2.3 \pm 1.8$ & $1.5 \pm 0.9$ & $<0.01^{* *}$ \\
AST $(\mathrm{U} / \mathrm{L})$ & $18.5 \pm 7.3$ & $17.7 \pm 3.8$ & $0.41^{* *}$ \\
ALT $(\mathrm{U} / \mathrm{L})$ & $15.9 \pm 9.9$ & $13.7 \pm 6.5$ & $0.14^{* *}$ \\
$\gamma$ GT $(\mathrm{U} / \mathrm{L})$ & $14.3 \pm 7.4$ & $12.4 \pm 4.5$ & $0.07^{* *}$ \\
TChol $(\mathrm{mg} / \mathrm{dl})$ & $185.0 \pm 41$ & $171.4 \pm 22$ & $<0.05^{*}$ \\
Trg $(\mathrm{mg} / \mathrm{dl})$ & $80.4 \pm 39.2$ & $67.1 \pm 33.9$ & $<0.05^{* *}$ \\
Testo $(\mathrm{nmol} / \mathrm{L})$ & $2.8 \pm 1.2$ & $1.7 \pm 0.5$ & $<0.001^{*}$ \\
fTesto $(\mathrm{pg} / \mathrm{ml})$ & $2,3 \pm 1,2$ & $1,7 \pm 0,5$ & $<0,01^{*}$ \\
SHBG $(\mathrm{nmol} / \mathrm{l})$ & $39.7 \pm 22.1$ & $51.0 \pm 27.8$ & $<0.05^{*}$ \\
FAI $\%$ & $9.6 \pm 7.0$ & $5.0 \pm 4.4$ & $<0.001^{*}$ \\
\hline
\end{tabular}

*unpaired t-test, **Mann-Whitney test, BMI: body mass index, HOMA-IR: homeostasis model assessment, AST: aspartate aminotransferase, ALT: alanine aminotransferase, $\gamma$ GT: $\gamma$-glutamyl transpeptidase, TChol: total cholesterol, Trig: triglycerides, Testo: total testosterone, fTesto: free testosterone, SHBG: sex hormone-binding globulin, FAI: free androgen index. Conversion factors for SI units are as follows: fTesto, $\mathrm{pmol} / \mathrm{l}=\mathrm{pg} / \mathrm{ml} \times 3.467$; Glucose, $\mathrm{mmol} / \mathrm{l}$ $=\mathrm{mg} / \mathrm{dl} \times 0.0555 ;$ Insulin, $\mathrm{pmol} / \mathrm{l}=\mu \mathrm{U} / \mathrm{ml} \times 7.175 ; \mathrm{TChol}, \mathrm{mmol} / \mathrm{l}=\mathrm{mg} / \mathrm{dl} \times$ $0.02586 ; \operatorname{Trg}, \mathrm{mmol} / \mathrm{l}=\mathrm{mg} / \mathrm{dl} \times 0.0113$

and $\gamma \mathrm{GT}$, were not significantly different between the two lean subgroups (Table 2). The prevalence of elevated ALT levels (>25 U/L) was $4.5 \%(2 / 44)$ in lean PCOS women and $5.9 \%(2 / 34)$ in lean controls $(\mathrm{p}=0.79)$. None of the lean patients or controls exhibited elevated $\gamma$ GT levels. In addition, none of the lean controls exhibited elevated insulin $(>12.5$ $\mu \mathrm{U} / \mathrm{ml}$ ), fasting glucose $>105 \mathrm{mg} / \mathrm{dl}$ or insulin resistance (HOMA-IR >2.5), the respective percentages in lean PCOS women being 6.8\% (3/44), 9.1\% (4/44) and $13.6 \%(6 / 44)$, compared to controls $(p=0.12$, $\mathrm{p}=0.07$ and $\mathrm{p}<0.05$, respectively).

Overweight/obese women with PCOS compared to overweight/obese controls had significantly higher fasting glucose $(\mathrm{p}<0.001)$, fasting insulin $(\mathrm{p}<0.05)$, HOMA-IR $(\mathrm{p}<0.05)$ and triglyceride values $(\mathrm{p}$ $<0.05$ ). Total cholesterol levels and AST serum levels were not significantly different between the two groups. However, serum levels of ALT and $\gamma$ GT were significantly higher in overweight/obese PCOS women compared to the respective values in overweight/obese controls $(p<0.05)$ (Table 2$)$. The percentage of overweight/obese PCOS with elevated liver enzymes (> $25 \mathrm{U} / \mathrm{L}$ ) was $20.5 \%$ (8/39) for ALT and $15.4 \%(6 / 39)$ for $\gamma \mathrm{GT}$, whereas the percentage in overweight/obese controls was 3\% (1/30) for both ALT and $\gamma$ GT (ALT: $\mathrm{p}<0.05, \gamma$-GT: $\mathrm{p}=0.10)$. The prevalence of insulin resistance, increased fasting glucose and increased fasting insulin in overweight/obese PCOS patients was $46.2 \%$ (18/39), 28.2\% (11/39) and 38.5\% (15/39), respectively, and in overweight/obese control subjects the respective values were $23.3 \%$ (18/39), $0 \%$ and $30 \%$ $(9 / 30)(p=0.051, p<0.01$ and $p=0.46$, respectively).

Overweight/obese PCOS patients had higher values of insulin resistance and exhibited elevated androgens, ALT and $\gamma$ GT levels when compared to lean PCOS patients [HOMA: $3.4 \pm 2.1$ vs $1.5 \pm 0.6 \mathrm{p}<0.001$ (unpaired t-test), FAI: $11.4 \pm 7.1$ vs $7.9 \pm 6.6$ (unpaired t-test), ALT: $17.7 \pm 12.2$ vs $12.5 \pm 5.8$ p $<0.01$ (MannWhitney test), $\gamma \mathrm{GT}$ : $17.9 \pm 9.1$ vs $11 \pm 3 \mathrm{p}<0.001$ (unpaired t-test)]. Overweight/obese controls had higher HOMA levels than their lean counterparts [ $2 \pm 1.1 \mathrm{vs}$ $1.1 \pm 0.5 \mathrm{p}<0.001$ (unpaired t-test)] with no difference in the levels of androgens or in liver enzymes.

In overweight/obese PCOS patients, $\gamma$ GT levels correlated with BMI (Pearson's $\mathrm{r}=0.35 \mathrm{p}<0.05$ ), fasting glucose (Pearson's $r=0.43 p<0.01$ ), fasting insulin (Pearson's $r=0.61 \mathrm{p}<0.001)$, HOMA (Pearson's $\mathrm{r}=0.6 \mathrm{p}<0.01)$ and $\operatorname{Trg}($ Pearson's $\mathrm{r}=0.43 \mathrm{p}<0.01)$ levels, while ALT correlated with insulin (Spearman's $\mathrm{r}=0.44 \mathrm{p}<0.01$ ), HOMA (Spearman's $\mathrm{r}=0.42 \mathrm{p}<0.05$ ) and Trg (Spearman's $r=0.35 \mathrm{p}<0,05)$. In lean PCOS patients, both $\gamma$ GT and ALT correlated positively with Trg levels (Pearson's $\mathrm{r}=0.31 \mathrm{p}<0.05$ and Pearson's $r=0.43 p<0.01$, respectively). In overweight/obese PCOS patients and in their respective controls, ALT levels were positively correlated with free androgen index (FAI) (Spearman's $r=0.25 \mathrm{p}<0.05$ and Pearson's $\mathrm{r}=0.77 \mathrm{p}<0.05$, respectively) and total testosterone levels (Spearman's $\mathrm{r}=0.33 \mathrm{p}<0.01$ and Pearson's $r=0.74 p<0.05$, respectively), while SHBG levels were negatively correlated with ALT (Spearman's $r=-0.23$ $\mathrm{p}<0.05$ and Pearson's $\mathrm{r}=-0.26 \mathrm{p}<0.05$, respectively) and $\gamma$ GT(Spearman's $r=-0.28 p<0.05$ and Spearman's $\mathrm{r}=-0.30 \mathrm{p}<0.05$, respectively). 
Table 2. Baseline anthropometric, biochemical and hormonal characteristics in overweight/obese and lean PCOS patients and in controls (mean \pm SD)

\begin{tabular}{|c|c|c|c|c|c|c|}
\hline \multirow[b]{2}{*}{ Variable } & \multicolumn{2}{|c|}{ Lean } & \multirow[b]{2}{*}{$P$} & \multicolumn{2}{|c|}{ Overweight/obese } & \multirow[b]{2}{*}{$P$} \\
\hline & $\operatorname{PCOS}(\mathrm{N}=44)$ & Controls $(\mathrm{N}=34)$ & & $\operatorname{PCOS}(\mathrm{N}=39)$ & Controls $(\mathrm{N}=30)$ & \\
\hline Age (years) & $23.6 \pm 4.5$ & $24.9 \pm 2.5$ & $0.14^{*}$ & $26.4 \pm 4.8$ & $28.5 \pm 3.8$ & $0.08^{*}$ \\
\hline $\operatorname{BMI}\left(\mathrm{kg} / \mathrm{m}^{2}\right)$ & $22.0 \pm 1.6$ & $21.4 \pm 1.6$ & $0.11^{*}$ & $30.1 \pm 2.8$ & $28.5 \pm 1.6$ & $0.07^{*}$ \\
\hline Glucose (mg/dl) & $88.6 \pm 11.2$ & $81.5 \pm 11.2$ & $<0.01^{*}$ & $100.1 \pm 13.9$ & $83.1 \pm 14.1$ & $<0.001^{*}$ \\
\hline Insulin $(\mu \mathrm{U} / \mathrm{ml})$ & $6.8 \pm 2.9$ & $5.4 \pm 2.0$ & $<0.05^{* *}$ & $13.6 \pm 2.9$ & $9.9 \pm 4.9$ & $<0.05^{*}$ \\
\hline HOMA-IR & $1.5 \pm 0.6$ & $1.1 \pm 0.5$ & $<0.01^{* *}$ & $3.4 \pm 2.1$ & $2.0 \pm 1.1$ & $<0.05^{*}$ \\
\hline $\operatorname{AST}(\mathrm{U} / \mathrm{L})$ & $18.3 \pm 8.5$ & $18.2 \pm 2.9$ & $0.93^{* *}$ & $18.8 \pm 5.7$ & $17.2 \pm 3.6$ & $0.18^{*}$ \\
\hline $\operatorname{ALT}(\mathrm{U} / \mathrm{L})$ & $12.5 \pm 5.8$ & $13.4 \pm 6.1$ & $0.52 *$ & $17.7 \pm 12.2$ & $14.1 \pm 7.0$ & $<0.05^{* *}$ \\
\hline$\gamma \mathrm{GT}(\mathrm{U} / \mathrm{L})$ & $11.0 \pm 3.0$ & $11.4 \pm 3.5$ & $0.61^{*}$ & $17.9 \pm 9.1$ & $13.4 \pm 5.2$ & $<0.05^{* *}$ \\
\hline TChol (mg/dl) & $179.5 \pm 44.6$ & $168.3 \pm 26.4$ & $0.20^{*}$ & $191.4 \pm 36.5$ & $174.7 \pm 31.3$ & $0.05^{*}$ \\
\hline $\operatorname{Trg}(\mathrm{mg} / \mathrm{dl})$ & $66.7 \pm 24.2$ & $56.2 \pm 14.0$ & $<0.05^{*}$ & $95.1 \pm 47.2$ & $79.4 \pm 44.6$ & $<0.05^{*}$ \\
\hline Testo (nmol/L) & $2.8 \pm 1.3$ & $1.9 \pm 0.4$ & $<0.001^{*}$ & $2.8 \pm 1.2$ & $1.8 \pm 0.3$ & $<0.001^{*}$ \\
\hline fTesto $(\mathrm{pg} / \mathrm{ml})$ & $2.1 \pm 1.0$ & $1.7 \pm 0.6$ & $0.05^{*}$ & $2.8 \pm 1.6$ & $1.6 \pm 0.4$ & $<0.05^{*}$ \\
\hline SHBG (nmol/l) & $49.1 \pm 25.7$ & $54.7 \pm 23.2$ & $0.34^{*}$ & $30.6 \pm 12.6$ & $46.9 \pm 31.9$ & $<0.01^{*}$ \\
\hline FAI $\%$ & $7.9 \pm 6.6$ & $4.1 \pm 2.1$ & $<0.01^{*}$ & $11.4 \pm 7.1$ & $6.0 \pm 5.9$ & $<0.01^{*}$ \\
\hline
\end{tabular}

* unpaired t-test, **Mann-Whitney test, BMI: body mass index, WHR: waist-to-hip ratio, HOMA-IR: homeostasis model assessment, AST: aspartate aminotransferase, ALT: alanine aminotransferase, $\gamma$ GT: $\gamma$-glutamyl transpeptidase, TChol: total cholesterol, Trig: triglycerides, Testo: total testosterone, fTesto: free testosterone, SHBG: sex hormone-binding globulin, FAI: free androgen index. Conversion factors for SI units are as follows: fTesto, $\mathrm{pmol} / \mathrm{l}=\mathrm{pg} / \mathrm{ml}$ x 3.467; Glucose, $\mathrm{mmol} / \mathrm{l}=\mathrm{mg} / \mathrm{dl} \times 0.0555 ; \mathrm{Insulin}, \mathrm{pmol} / \mathrm{l}=$ $\mu \mathrm{U} / \mathrm{ml} \times 7.175 ; \mathrm{TChol}, \mathrm{mmol} / \mathrm{l}=\mathrm{mg} / \mathrm{dl} \times 0.02586 ; \mathrm{Trg}, \mathrm{mmol} / \mathrm{l}=\mathrm{mg} / \mathrm{dl} \times 0.0113$

Stepwise multiple regression analysis revealed that BMI and HOMA index have the highest impact on the variability of liver enzymes. The R-Squared statistic indicates that the two predictors explain $35.4 \%$ of the variability for $\gamma$ GT and $17.7 \%$ for ALT, respectively. The equations of the fitted models are $[\gamma \mathrm{GT}$ $=0.693733+0.36661 \times \mathrm{BMI}+1.65396 \times \mathrm{HOMA}]$ $($ F-ratio $=36.58, \mathrm{p}<0.001)(\mathrm{BMI}: \mathrm{p}<0.01, \mathrm{HOMA}$ : $\mathrm{p}<0.001)$ and $[\mathrm{ALT}=2.39961+0.377264 \times \mathrm{BMI}+$ $1.59366 \times$ HOMA] $($ F-ratio $=14.39, \mathrm{p}<0.001)(\mathrm{BMI}:$ $\mathrm{p}<0.05$, HOMA: $\mathrm{p}<0.01)$.

\section{DISCUSSION}

In this cohort of premenopausal women it was demonstrated that overweight/obese women with PCOS have elevated serum levels of ALT and $\gamma$ GT as compared with the respective levels in the control groups of comparable age and BMI. However, we did not identify any significant difference in serum liver enzymes between lean PCOS and lean controls and between the total PCOS and total control population. The lean PCOS group exhibited higher insulin resist- ance expressed by HOMA-IR than the respective control group, but the absolute level of HOMA-IR in lean PCOS is below the accepted cutoff level for insulin resistance. These findings suggest that obesity and the severity of insulin resistance appear to have an additive effect on elevated liver enzymes. This is also shown by the positive correlation of HOMA-IR and BMI with $\gamma$ GT and ALT and is further supported by the outcome of multiple regression analysis indicating that HOMA-IR and BMI and not the PCOS status appear to be the major determinants of both $\gamma$-GT and ALT in our cohort.

In most previous studies, in which BMI and age matched controls were not included, increased serum aminotransferases, and especially ALT, and an increased prevalence of NAFLD were found in PCOS women. ${ }^{21,22,25}$ Their conclusions, however, of an increased prevalence of hypertransaminasemia and of NAFLD in PCOS were based on comparisons with the respective prevalence rates reported in the general population. The mild metabolic derangement as well as the relatively lower mean BMI in 
the subjects of our study might account for the lower levels of serum aminotransferases as compared to the previously reported levels in PCOS women. ${ }^{21,22}$ In other words, our patient population consisted of subjects without overt metabolic abnormalities; the triglyceride levels in PCOS women were higher than in controls but still remained within normal limits. In previous studies, lean PCOS patients did not exhibit evidence of NAFLD or elevated liver enzymes in respect to lean controls, ${ }^{23}$ whereas obese PCOS women were shown to have significantly elevated liver enzymes in comparison to controls, ${ }^{24}$ findings that are in agreement with our results, although to a moderate degree since our study population was relatively less obese.

Insulin resistance was present in $41.6 \%$ of the overweight/obese PCOS women and $23.3 \%$ in overweight/obese controls, whereas it was present in $13.6 \%$ of the lean PCOS women and in none of the lean controls. The values of hepatic enzymes ( $\gamma \mathrm{GT}, \mathrm{ALT})$ in overweight/obese PCOS women were positively correlated with BMI, HOMA-IR and triglycerides levels, the latter being an indirect indicator of insulin resistance.

Since ultrasound findings usually precede liver biochemistry abnormalities, ${ }^{25}$ the fact that a previous study in lean PCOS has shown positive $\mathrm{u} / \mathrm{s}$ findings compatible with fatty liver with normal liver enzymes should not be considered as contradicting our findings. In that study, normal serum aminotransferases coexisted with $\mathrm{u} / \mathrm{s}$ findings compatible with NAFLD in a significant proportion of lean and obese patients with PCOS. ${ }^{25}$ In the same study, patients with PCOS were subdivided into various BMI groups and were investigated for NAFLD, but without a control group. An additional confounder was the fact that $64 \%$ of patients were receiving some form of pharmacotherapy, namely metformin and/or oral contraceptives, at the time of evaluation. ${ }^{25}$ Additionally, subjects included in this and other studies ${ }^{24,25}$ differed from our study population in ethnic composition.

As mentioned above, all lean patients had a normal lipid profile which was comparable to that in controls, with the exception of serum triglycerides, which nevertheless remained within normal limits. Although fasting serum insulin and glucose levels were higher in lean patients than in lean controls, only 3 out of 38 lean patients had marginal fasting hyperinsulinemia. Moreover, none of the lean PCOS patients had fasting hyperglycemia. This may explain the lack of detectable differences in serum liver enzymes in lean patients in our study compared with their lean controls.

The positive correlation of ALT with total testosterone and FAI, shown for the first time, may indicate an additional adverse effect of hyperandrogenemia on liver biochemistry ${ }^{30}$ in PCOS. This effect is further demonstrated by the finding that overweight/obese PCOS patients were more insulin resistant and had higher ALT, $\gamma$ GT and FAI levels compared with lean PCOS, whereas overweight/obese controls, despite being more insulin resistant than their lean counterparts, did not exhibit higher liver enzymes. This association between androgen and liver enzyme levels could be an indirect reflection of insulin resistance and compensatory hyperinsulinemia, which may stimulate ovarian androgen production in PCOS women ${ }^{31,32}$ and also inhibit hepatic production of SHBG ${ }^{33}$ further increasing FAI.

Serum aminotransferase levels should not be considered as accurate diagnostic indicators of NAFLD. The complete histological spectrum of NAFLD was reported in patients with normal ALT activity ${ }^{34}$ and a falsely normal ALT can be seen in $30 \%$ of individuals with NASH. ${ }^{35}$ Conversely, increased ALT not associated with fatty liver has been observed in obese subjects. ${ }^{36}$

On the other hand, numerous studies have shown that NAFLD comprises by far the commonest histological diagnosis in subjects with unexplained elevations of liver enzymes, after excluding known causes of liver dysfunction. ${ }^{37-40}$ In particular, ALT might be a preclinical marker of NAFLD. A slightly increased serum ALT concentration might reflect subclinical (or ultrasonographically undetectable) early fatty changes in the liver (hepatic steatosis), which precedes the overt NAFLD. ${ }^{41}$ In a large healthy, non-diabetic population, increasing ALT concentration, even within the reference range, was an independent predictor of NAFLD assessed by ultrasonography, irrespective of potential confounders, including BMI, alcohol consumption, CRP, HOMA-IR, fasting glucose, lipids 
and blood pressure. ${ }^{41}$ This relationship remained even after adjustment for diabetes and weight gain.

Our findings in lean and overweight/obese women with PCOS may have a dual importance. Since overweight/obese women with PCOS appear to be more prone to develop NAFLD, screening for NAFLD in obese and/or overweight PCOS women is most likely indicated. Serum liver biochemistry should be determined in these women in combination with ultrasonographic evaluation for the detection of fatty liver infiltration. NAFLD is no longer considered to be devoid of long-term health implications. Although uncomplicated hepatic steatosis (NAFL) may be a benign condition, it has the potential to progress to $\mathrm{NASH}$, which is an established cause of 'cryptogenic' cirrhosis. ${ }^{42}$ Using both liver function tests and ultrasound, NASH may be diagnosed earlier and prompt an early stage approach that could reduce its impact on the patient's health in conjunction with lifestyle modifications ${ }^{43}$ and/or insulin-sensitizing agents. ${ }^{44}$ Both NAFLD and elevated serum liver enzymes - independently of NAFLD-emerge as novel components of the metabolic syndrome and may be considered as risk factors for type 2 diabetes and cardiovascular disease. Additionally, neither insulin resistance nor hyperandrogenemia were found to alter the hepatic profile in the subgroup of lean PCOS women. Obesity and insulin resistance appear to be the aggravating factors for disturbed hepatic biochemical profile in women with PCOS compared to controls.

In conclusion, it is advisable to look for NAFLD and abnormal hepatic liver enzymes mainly in overweight/obese PCOS women since higher BMI and insulin resistance appear to be the major factors influencing liver enzymes elevation.

\section{REFERENCES}

1. Diamanti-Kandarakis E, Kouli CR, Bergiele AT, et al, 1999 A survey of the polycystic ovary syndrome in the Greek island of Lesbos: hormonal and metabolic profile. J Clin Endocrinol Metab 84: 4006-4011.

2. Dunaif A, Segal KR, Futterweit W, Dobrjansky A, 1989 Profound peripheral insulin resistance, independent of obesity, in polycystic ovary syndrome. Diabetes 38: 1165-1174.

3. Salley KE, Wickham EP, Cheang KI, Essah PA, Karjane NW, Nestler JE, 2007 Glucose intolerance in polycystic ovary syndrome - a position statement of the Androgen Excess Society. J Clin Endocrinol Metab 92: 45464556.

4. Diamanti-Kandarakis E, Papavassiliou AG, Kandarakis SA, Chrousos GP, 2007 Pathophysiology and types of dyslipidemia in PCOS. Trends Endocrinol Metab 18: 280-285.

5. Azziz R, 2006 How prevalent is metabolic syndrome in women with polycystic ovary syndrome? Nat Clin Pract Endocrinol Metab 2: 132-133.

6. Norman RJ, Dewailly D, Legro RS, Hickey TE, 2007 Polycystic ovary syndrome. Lancet 370: 685-697.

7. Diamanti-Kandarakis E, Paterakis T, Kandarakis HA, 2006 Indices of low-grade inflammation in polycystic ovary syndrome. Ann N Y Acad Sci 1092: 175-186.

8. Diamanti-Kandarakis E, Piperi C, Kalofoutis A, Creatsas G, 2005 Increased levels of serum advanced glycation end-products in women with polycystic ovary syndrome. Clin Endocrinol (Oxf) 62: 37-43.

9. Diamanti-Kandarakis E, 2008 Polycystic ovarian syndrome: pathophysiology, molecular aspects and clinical implications. Expert Rev Mol Med 10:e3.

10. Agarwal N, Sharma BC, 2005 Insulin resistance and clinical aspects of non-alcoholic steatohepatitis (NASH). Hepatol Res 33: 92-96.

11. Ahima RS, 2007 Insulin resistance: cause or consequence of nonalcoholic steatohepatitis? Gastroenterology 132: 444-446.

12. Angelico F, Del BM, Conti R, et al, 2005 Insulin resistance, the metabolic syndrome, and nonalcoholic fatty liver disease J Clin Endocrinol Metab 90: 1578-1582.

13. Farrell GC, Larter CZ, 2006 Nonalcoholic fatty liver disease: from steatosis to cirrhosis. Hepatology 43: S99S112.

14. Clark JM, Brancati FL, Diehl AM, 2003 The prevalence and etiology of elevated aminotransferase levels in the United States. Am J Gastroenterol 98: 960-967.

15. Cortez-Pinto H, de Moura MC, Day CP, 2006 Nonalcoholic steatohepatitis: from cell biology to clinical practice. J Hepatol 44: 197-208.

16. Schindhelm RK, Diamant M, Dekker JM, Tushuizen ME, Teerlink T, Heine RJ, 2006 Alanine aminotransferase as a marker of non-alcoholic fatty liver disease in relation to type 2 diabetes mellitus and cardiovascular disease. Diabetes Metab Res Rev 22: 437-443.

17. Adams LA, Angulo P, Lindor KD, 2005 Nonalcoholic fatty liver disease. CMAJ 172: 899-905.

18. Targher G, 2007 Non-alcoholic fatty liver disease, the metabolic syndrome and the risk of cardiovascular disease: the plot thickens. Diabet Med 24: 1-6.

19. Devers MC, Campbell S, Shaw J, Zimmet P, Simmons D, 2008 Should liver function tests be included in definitions of metabolic syndrome? Evidence from the association between liver function tests, components of metabolic syndrome and prevalent cardiovascular disease. Diabet Med 25: 523-529. 
20. Sattar N, Wannamethee SG, Forouhi NG, 2008 Novel biochemical risk factors for type 2 diabetes: pathogenic insights or prediction possibilities? Diabetologia 51: 926-940.

21. Schwimmer JB, Khorram O, Chiu V, Schwimmer WB, 2005 Abnormal aminotransferase activity in women with polycystic ovary syndrome. Fertil Steril 83: 494-497.

22. Setji TL, Holland ND, Sanders LL, Pereira KC, Diehl AM, Brown AJ, 2006 Nonalcoholic steatohepatitis and nonalcoholic fatty liver disease in young women with polycystic ovary syndrome. J Clin Endocrinol Metab 91: 1741-1747.

23. Markou A, Androulakis II, Mourmouris C, et al, 2009 Hepatic steatosis in young lean insulin resistant women with polycystic ovary syndrome Fertil Steril: Jan 24 (in press).

24. Cerda C, Perez-Ayuso RM, Riquelme A, et al, 2007 Nonalcoholic fatty liver disease in women with polycystic ovary syndrome. J Hepatol 47: 412-417.

25. Gambarin-Gelwan M, Kinkhabwala SV, Schiano TD, Bodian C, Yeh HC, Futterweit W, 2007 Prevalence of nonalcoholic fatty liver disease in women with polycystic ovary syndrome. Clin Gastroenterol Hepatol 5: 496501.

26. Prati D, Taioli E, Zanella A, et al, 2002 Updated definitions of healthy ranges for serum alanine aminotransferase levels. Ann Intern Med 137: 1-10.

27. Zawadski JK, Dunaif A, 1992 Diagnostic criteria for polycystic ovary syndrome: towards a rational approach. Polycystic ovary syndrome: Blackwell Scientific Publications Inc, Boston; pp, 377-384.

28. Diamanti-Kandarakis E, Paterakis T, Alexandraki K, et al, 2006 Indices of low-grade chronic inflammation in polycystic ovary syndrome and the beneficial effect of metformin. Hum Reprod 21: 1426-1431.

29. Bergman RN, Prager R, Volund A, Olefsky JM, 1987 Equivalence of the insulin sensitivity index in man derived by the minimal model method and the euglycemic glucose clamp. J Clin Invest 79: 790-800.

30. Maffei L, Murata Y, Rochira V, et al, 2004 Dysmetabolic syndrome in a man with a novel mutation of the aromatase gene: effects of testosterone, alendronate, and estradiol treatment. J Clin Endocrinol Metab 89: 61-70.

31. Nestler JE, Barlascini CO, Matt DW, et al, 1989 Suppression of serum insulin by diazoxide reduces serum testosterone levels in obese women with polycystic ovary syndrome. J Clin Endocrinol Metab 68: 1027-1032.

32. Nestler JE, Jakubowicz DJ, de Vargas AF, Brik C, Quintero N, Medina F, 1998 Insulin stimulates testosterone biosynthesis by human thecal cells from women with polycystic ovary syndrome by activating its own receptor and using inositolglycan mediators as the signal transduction system. J Clin Endocrinol Metab 83: 2001-2005.

33. Nestler JE, Powers LP, Matt DW, et al, 1991 A direct effect of hyperinsulinemia on serum sex hormone-binding globulin levels in obese women with the polycystic ovary syndrome. J Clin Endocrinol Metab 72: 83-89.

34. Kunde SS, Lazenby AJ, Clements RH, Abrams GA, 2005 Spectrum of NAFLD and diagnostic implications of the proposed new normal range for serum ALT in obese women. Hepatology 42: 650-656.

35. Bloomgarden ZT, 2007 Nonalcoholic fatty liver disease and insulin resistance in youth. Diabetes Care 30: 16631669.

36. Sakugawa H, Nakayoshi T, Kobashigawa K, et al, 2004 Alanine aminotransferase elevation not associated with fatty liver is frequently seen in obese Japanese women. Eur J Clin Nutr 58: 1248-1252.

37. Daniel S, Ben-Menachem T, Vasudevan G, Ma CK, Blumenkehl M, 1999 Prospective evaluation of unexplained chronic liver transaminase abnormalities in asymptomatic and symptomatic patients. Am J Gastroenterol 94: 30103014.

38. Hultcrantz R, Glaumann H, Lindberg G, Nilsson LH, 1986 Liver investigation in 149 asymptomatic patients with moderately elevated activities of serum aminotransferases. Scand J Gastroenterol 21: 109-113.

39. Mathiesen UL, Franzen LE, Fryden A, Foberg U, Bodemar G, 1999 The clinical significance of slightly to moderately increased liver transaminase values in asymptomatic patients. Scand J Gastroenterol 34: 8591.

40. Skelly MM, James PD, Ryder SD, 2001 Findings on liver biopsy to investigate abnormal liver function tests in the absence of diagnostic serology. J Hepatol 35: 195-199.

41. Chang Y, Ryu S, Sung E, Jang Y, 2007 Higher concentrations of alanine aminotransferase within the reference interval predict nonalcoholic fatty liver disease. Clin Chem 53: 686-692.

42. Clark JM, Diehl AM, 2003 Nonalcoholic fatty liver disease: an underrecognized cause of cryptogenic cirrhosis. JAMA 289: 3000-3004.

43. Brown AJ, Tendler DA, McMurray RG, Setji TL, 2005 Polycystic ovary syndrome and severe nonalcoholic steatohepatitis: beneficial effect of modest weight loss and exercise on liver biopsy findings. Endocr Pract 11: 319-324.

44. Preiss D, Sattar N, Harborne L, Norman J, Fleming R, 2008 The effects of 8 months of metformin on circulating GGT and ALT levels in obese women with polycystic ovarian syndrome. Int J Clin Pract 62: 1337-1343. 